\title{
Emerging treatments in the management of schizophrenia - focus on sertindole
}

This article was published in the following Dove Press journal:

Drug Design, Development and Therapy

23 August 2010

Number of times this article has been viewed

\section{Maria Rosaria A Muscatello \\ Antonio Bruno \\ Gianluca Pandolfo \\ Umberto Micò \\ Salvatore Settineri \\ Rocco Zoccali}

Section of Psychiatry, Department of Neurosciences, Psychiatric and Anaesthesiological Sciences, University of Messina, Italy
Correspondence: Rocco Zoccali Department of Neurosciences, Psychiatric and Anesthesiological Sciences, Policlinico Universitario Via Consolare Valeria - Messina 98 I 25 Italy Tel +090 222I 2092

Fax +090695 I36

Email rocco.zoccali@unime.it

\begin{abstract}
The antipsychotic treatment of schizophrenia is still marked by poor compliance, and drug discontinuation; the development of more effective and safer drugs still remains a challenge. Sertindole is a second-generation antipsychotic with high affinity for dopamine $\mathrm{D}_{2}$, serotonin $5-\mathrm{HT}_{2 \mathrm{~A}}, 5-\mathrm{HT}_{2 \mathrm{C}}$, and $\alpha_{1}$-adrenergic receptors, and low affinity for other receptors. Sertindole undergoes extensive hepatic metabolism by the cytochrome P450 isoenzymes CYP2D6 and CYP3A4 and has an elimination half-life of approximately three days. In controlled clinical trials sertindole was more effective than placebo in reducing positive and negative symptoms, whereas it was as effective as haloperidol and risperidone against the positive symptoms of schizophrenia. The effective dose-range of sertindole is $12-20 \mathrm{mg}$, administered orally once daily. The most common adverse events are headhache, insomnia, rhinitis/nasal congestion, male sexual dysfunction, and moderate weight gain, with few extrapyramidal symptoms and metabolic changes. Sertindole is associated with corrected QT interval prolongation, with subsequent risk of serious arrythmias. Due to cardiovascular safety concerns, sertindole is available as a second-line choice for patients intolerant to at least one other antipsychotic agent. Further clinical studies, mainly direct "head-to-head" comparisons with other second-generation antipsychotic agents, are needed to define the role of sertindole in the treatment of schizophrenia.
\end{abstract}

Keywords: antipsychotics, pharmacology, pharmacokinetics, efficacy, safety

\section{Introduction}

Schizophrenia, a severe and debilitating mental illness affecting approximately $1 \%$ of the population ${ }^{1}$ is a heterogeneous disorder characterized by positive and negative symptoms and often associated with cognitive impairment and affective, mainly depressive, symptoms. The course of schizophrenia is chronic, with relapses in psychotic episodes, disturbed cognitive functioning, poor quality of life and social decline. Psychopharmacological treatments have significantly modified the longitudinal course and outcome of schizophrenia. However, according to Tamminga and Holcomb, ${ }^{2}$ only $10 \%-20 \%$ of patients with schizophrenia show a good outcome, recovering to pre-illness levels of functioning. Another $15 \%-20 \%$ show a poor outcome and are considered treatment resistant. The middle group presents residual symptoms in several domains of schizophrenia, such as cognitive, psychotic and affective symptoms, and impaired socio-occupational functioning despite medications. Nevertheless, antipsychotic medication is the primary intervention for the stabilization of acute psychotic episodes and the prevention of recurrences and relapses in patients with schizophrenia. A key feature for therapeutic success is drug safety 
and tolerability; an incomplete response is often the result of poor tolerability because side effects can affect treatment compliance. Safety and tolerability issues range from specific, fairly simple adverse events (AEs) to more severe, life-threatening conditions such as agranulocytosis, acute diabetes and cardiac arrhythmia.

"Atypical" or second-generation antipsychotics, were initially said to differ from typical antipsychotics in that they do not cause movement disorders (catalepsy) in rats at clinically effective doses. ${ }^{3}$ Over the past decade, they have become the treatment of choice for schizophrenia in many countries, due to the perception of a more favorable tolerability profile, thus raising major hopes of superior effects in a number of areas such as compliance, negative symptoms, cognitive functioning, movement disorders, and quality of life. According to treatment guidelines, ${ }^{4,5}$ second-generation antipsychotics include drugs such as amisulpride, aripiprazole, clozapine, olanzapine, quetiapine, risperidone, sertindole, ziprasidone and zotepine. Besides dopamine receptor antagonism, second-generation antipsychotics have an additional range of binding profile at other receptor sites, including those for serotonin $\left(5-\mathrm{HT}_{1 \mathrm{~A}, 2 \mathrm{~A}, 2 \mathrm{C}, 3,6,7}\right)$, and norepinephrine $\left(\alpha_{1}\right.$ and $\left.\alpha_{2}\right){ }^{6}$ Second-generation antipsychotics are characterized by a low propensity to induce acute extrapyramidal symptoms and tardive dyskinesia and, with the exception of risperidone and amisulpride, by a weak potential to cause hyperprolactinemia. ${ }^{7}$ Nevertheless, the safety advantages of the atypical drugs have been questioned, as their use is associated with differential risk of metabolic effect, such as weight gain, lipid dysregulation and hyperglycemia, ${ }^{8}$ and cardiovascular adverse events, particularly prolongation of heart-rate corrected QT interval (QTc) of the electrocardiogram (ECG). ${ }^{9}$

The Clinical Antipsychotic Trials of Intervention Effectiveness (CATIE) was the first objective comparison of multiple antipsychotic drugs carried out with patients in settings that were representative of real-world treatment settings for people with schizophrenia. The trial showed that, in patients with chronic schizophrenia, antipsychotic treatment was marked by poor compliance, drug discontinuation and frequent switching due to lack of efficacy. Poor compliance equally affected both conventional and atypical antipsychotics, with the exception of olanzapine. Furthermore, it confirmed the association between metabolic disorders and atypical antipsychotics. ${ }^{10,11}$

Sertindole is an atypical antipsychotic that was initially marketed within the European Union between 1996 and 1997 for the treatment of schizophrenia. However, data from the Adverse Drug Reaction On-line Information Tracking (ADROIT) database in the United Kingdom subsequently suggested an increase in cardiovascular mortality with sertindole compared with other atypical antipsychotics. By the end of November 1998, the Committee on Safety of Medicines (CSM), and the Medicine Control Agency (MCA) had been notified of 36 suspected adverse drug reactions with a fatal outcome and 13 reports of serious, even nonfatal arrythmias in patients treated with sertindole. This potential effect, ascribed to the QT-interval prolongation induced by sertindole, led to the suspension of all European Union marketing authorizations in 1998, pending a full evaluation of its risk-benefit profile. Since then, nonclinical data showed that QT-interval prolongation was not related with ventricular arrhythmias. ${ }^{12-14}$ Moreover, results from epidemiological studies failed to confirm the ADROIT signal and did not indicate an excess of overall mortality with sertindole relative to other recently developed antipsychotics. ${ }^{15-17}$

Based on this evidence, the Committee for Medicinal Products for Human Use (CHMP) opted to reintroduce sertindole for use in Europe under certain restrictions, such as on a named-patient/compassionate use program (NPU) in post-marketing surveillance studies. Sertindole was finally approved by the European Commission and made available in the European market in 2006.

This article analyzes the pharmacological properties, clinical efficacy, tolerability and safety profile of sertindole in patients with schizophrenia.

\section{Data sources and selection of studies}

Using the keywords "sertindole", and "schizophrenia", a literature search was undertaken using the international database PubMed, to find all the studies published in English. Additional references were identified from the reference lists of published articles. Data were also collected from the product labelling. Searches were last updated on March 5, 2010 .

\section{Pharmacological profile}

Sertindole is a phenylindole derivative (1-[2-[4-[5-chloro1-(4-fluorophenyl)-1H-indol-3-yl]-1-piperidinyl] ethyl-2imidazolidinone). In vitro studies showed that sertindole exerts a potent antagonism at serotonin $5-\mathrm{HT}_{2 \mathrm{~A}}, 5-\mathrm{HT}_{2 \mathrm{C}}$, dopamine $\mathrm{D}_{2}$, and $\alpha_{1}$-adrenergic receptors, with binding affinities of $0.20,0.51,0.45$, and $1.4 \mathrm{nmol} /$, respectively, whereas it has a low affinity for cholinergic muscarinic, histamine $H_{1}$, and $\alpha_{2}$-adrenergic receptors..$^{3,18,19}$ Moreover, 


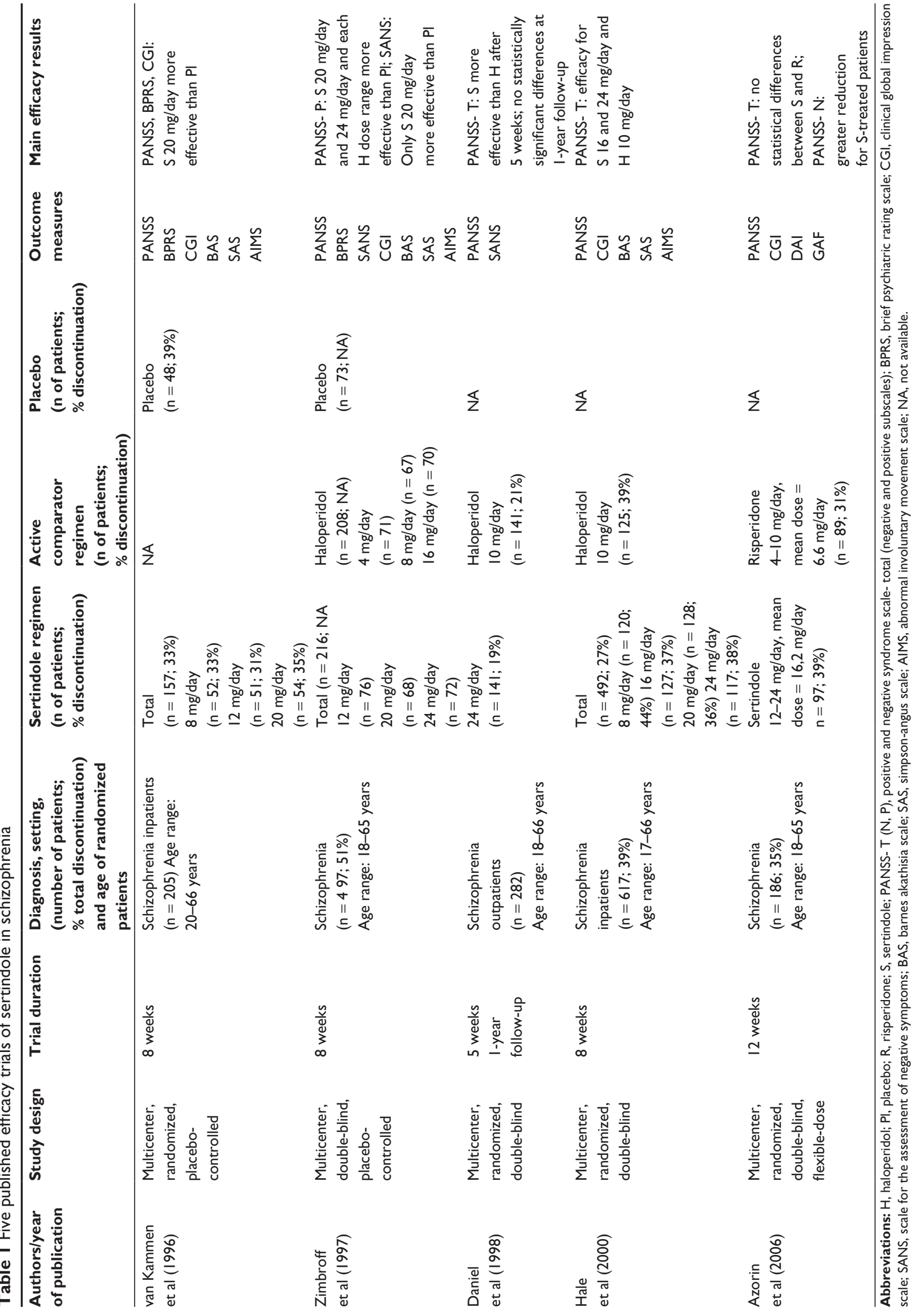


sertindole has high 5-HT6 receptor affinity (in vitro binding affinity $=9.13 \mathrm{pKi}$ value, $-\log M) .{ }^{20}$ Combined dopamine $\mathrm{D}_{2}$ and serotonin $5-\mathrm{HT}_{2 \mathrm{~A}}$ receptor antagonism is a feature common to most atypical antipsychotics and is thought to contribute to their therapeutic effect on schizophrenic symptoms. ${ }^{21-23}$ Results from a positron emission tomography (PET) study on healthy subjects showed that sertindole up to $12 \mathrm{mg} /$ day induced a $5-\mathrm{HT}_{2 \mathrm{~A}}$ receptor occupancy of approximately $100 \%$, thus confirming preclinical data on sertindole receptor affinity. ${ }^{24}$ Biochemical, electrophysiological and behavioral studies in animal models showed that sertindole selectively inhibits mesolimbic rather than nigrostriatal dopaminergic activity, thus suggesting a low potential to induce extrapyramidal symptoms (EPS) when compared with conventional antipsychotics. ${ }^{25-27}$ Another PET study on four patients affected by schizophrenia after 6-8 weeks of treatment with sertindole up to $20 \mathrm{mg} /$ day, aimed to investigate the $\mathrm{D}_{2}$ receptor occupancy in striatal and extra-striatal regions, evidenced that sertindole was effective at relatively low striatal $\mathrm{D}_{2}$ receptor occupancy $(52 \%-68 \%)$, thus suggesting a low risk for EPS. ${ }^{28}$

The receptor-affinity profile of sertindole, particularly its weak antagonism at cholinergic muscarinic and histaminergic $\mathrm{H}_{1}$ receptors, provides an explanation for its beneficial effect on cognitive functioning. In animal models, sertindole, and also risperidone and clozapine, but not haloperidol, were observed to reverse cognitive deficits induced by repeated PCP administration measured by the Morris water maze. ${ }^{29}$ Moreover, differently from risperidone, olanzapine, and clozapine, sertindole induced a significant reversal of impairment in extradimensional set shifting and attentional shifting following subchronic PCP administration, ${ }^{30-32}$ and improves subchronic PCP-induced reversal learning and episodic memory deficits in rodents. ${ }^{33}$

\section{Pharmacokinetics}

Sertindole is slowly absorbed by the gastrointestinal tract, reaching peak concentrations within ten hours after oral doses in healthy subjects and renally impaired patients. The relative bioavailability of the tablet formulation is about $75 \%$ and absorption is significantly affected by food or antacids. ${ }^{34-36}$ Over the dose range of 4-16 $\mathrm{mg} /$ day, dose-proportional increases in the maximum plasma concentrations (Cmax) [from 2.0 to $9.1 \mathrm{ng} / \mathrm{mL}$ ] and the area under the plasma concentration-time curve (AUC from 170 to $572 \mathrm{ng} \cdot \mathrm{h} / \mathrm{mL}$ ) of sertindole have been documented. ${ }^{37}$ Once absorbed, sertindole readily distributes in tissues, as evidenced by the large volume distribution of approximately $20 \mathrm{~L} / \mathrm{kg}$; its plasma protein binding, mainly to albumin and $\alpha_{1}$-acid glycoprotein, is $99.5 \%{ }^{38}$ Sertindole readily penetrates both the blood-brain and placental barriers because of its basic lipophilic property; however, differently from other antipsychotics, it is not known if sertindole acts as a substrate or an inhibitor of the efflux transporter P-glycoproteine which is highly expressed in the brain, liver, kidney, and gut. ${ }^{39,40}$ Sertindole undergoes extensive metabolism in the liver by the cytochrome P450 isoenzymes CYP2D6 and CYP3A4, to two main metabolites, dehydrosertindole (via oxidation) and norsertindole (via N-dealkylation) with negligible effects in humans. Genetic polymorphism of the CYP2D6 isoform leads to a moderate interindividual variation in the pharmacokinetics of sertindole, with reduction in sertindole clearance up to $67 \%$ and plasma sertindole concentrations 2-3 times higher in poor metabolizers (PM, up to $10 \%$ of the general population) when compared with extensive metabolizers (EM). Sertindole and its metabolites are excreted slowly, mainly in the feces with a minor amount appearing in the urine. Its mean terminal half-life is 53-102 hours, as evidenced in single- and multiple-dose studies in healthy subjects; ${ }^{34,35}$ therefore, steady-state is reached within 2-3 weeks. The relatively long half-life of sertindole in comparison to other atypical antipsychotics may represent a potential benefit in clinical practice and patient compliance. Neither gender nor age seem to affect the pharmacokinetics of sertindole. Nevertheless, slower titration and lower maintenance dose may be appropriate in elderly patients who are especially sensitive to the hypotensive effects of the drug. ${ }^{35}$ Dosage adjustment is not necessary in patients with mild, moderate, and severe renal impairment, ${ }^{36}$ whereas a dose reduction is required in hepatically impaired patients, as the clearance of sertindole decreases in this population.

\section{Drug interactions}

The importance of CYP2D6 and CYP3A4 in sertindole clearance is underlined by its interactions with potent inhibitors of these isoenzymes. Concomitant treatment with fluoxetine, paroxetine and quinidine (CYP2D6 inhibitors) and erythromycin, ketoconazole and indinavir (CYP3A4 inhibitors), may raise plasma sertindole concentrations. Nevertheless, oral coadministration of erythromycin, $250 \mathrm{mg} 4$ times/day, caused a slight increase in plasma AUC of sertindole given as a single $4 \mathrm{mg}$ dose to healthy subjects. ${ }^{41}$ On the other hand, plasma sertindole concentrations may decrease during concomitant treatment with CYP inducers such as carbamazepine, rifampicin, phenobarbital, and phenytoin. Recent in vitro biochemical evidence has showed that sertindole, 
which has the lowest inhibitory constant $(\mathrm{Ki}=25 \mu \mathrm{M})$ among the tested drugs (phenotiazines, haloperidol, and risperidone), should not inhibit the activity of CYP2C6 when administered in vivo. ${ }^{42}$ Thus, it seems unlikely that sertindole may interfere in the biotransformation of concomitantly given medications. Formal kinetic studies in healthy volunteers have showed that the pharmacokinetic parameters of alprazolam and terfenadine where not significantly affected by the concomitant treatment with sertindole, administered at daily doses of $12 \mathrm{mg}$ and $20 \mathrm{mg}$, respectively. ${ }^{43,44}$

Because of the effect on QTc interval, sertindole is contraindicated in combination with drugs known to significantly increase the QTc interval, such as some antihistamines (terfenadine and astemizole), some antiarrhythmics (amiodarone and quinidine), quinolone antibiotics, and many antipsychotics and antidepressants. ${ }^{38}$

\section{Efficacy \\ Positive and negative symptoms}

The efficacy of sertindole in patients with schizophrenia has been established in five randomized, double-blind clinical trials (see Table 1), without considering results from smaller studies presented as case reports, posters, and proceedings of congresses. These studies included one placebo-controlled trial, ${ }^{45}$ three comparisons with haloperidol, ${ }^{46-48}$ and one with risperidone. ${ }^{49}$ Of the five main published sertindole trials, only one evaluated the long-term outcome. ${ }^{48}$

A randomized, controlled, dose-ranging trial, involving 205 participants, evaluated the efficacy of sertindole, administered at the dosage of 8-20 mg/day for 6-7 weeks, in the treatment of schizophrenia. Sertindole at the dose of $20 \mathrm{mg} /$ day was more effective than placebo in reducing Positive and Negative Syndrome Scale (PANSS), and brief Psychiatric Rating Scale (BPRS) total scores. At the end of the trial, significantly more patients treated with sertindole $20 \mathrm{mg}$ /day than with placebo were "very much improved" (20\% vs $3 \% ; P<0.05)$ considering Clinical Global Impression (CGI) scores. No statistically significant differences were found on the outcome measures for sertindole at doses of 8 and $12 \mathrm{mg} /$ day. ${ }^{45}$ Sertindole has been compared to haloperidol in two short-term ${ }^{46,47}$ and one long-term studies. ${ }^{48}$

In a multicenter, double-blind, placebo-controlled study by Zimbroff et al, ${ }^{46} 497$ hospitalized patients with schizophrenia and active psychosis were randomized to receive sertindole 12,20 or $24 \mathrm{mg} /$ day, haloperidol 4,8 or $16 \mathrm{mg} /$ day, or placebo for an 8-week period. Measures of efficacy included the Positive and Negative Syndrome Scale (PANSS), the Scale for the Assessment of Negative Symptoms (SANS), the Clinical Global Impression (CGI) scale, and the Brief Psychiatric Rating Scale (BPRS). Three rating scales were used to assess EPS, as well as the occurrence of adverse events. Both sertindole and haloperidol were significantly more effective than placebo in the treatment of psychosis, as documented by improvements from baseline in PANSS and BPRS total scores at the end of the trial. Sertindole and haloperidol were equally effective, with the best results seen with the $20 \mathrm{mg} /$ day dose of sertindole and the $8 \mathrm{mg} /$ day dose of haloperidol.

Another multicenter, double-blind phase III trial, ${ }^{47}$ involved 617 patients who were randomized to receive sertindole $8,16,20$ or $24 \mathrm{mg} /$ day or haloperidol $10 \mathrm{mg}$ /day for 8 weeks. The sertindole $8 \mathrm{mg} /$ day dose was chosen as a 'pseudo-placebo'. A significant reduction in PANSS total score was observed with sertindole $16 \mathrm{mg}$ /day (by 23.8 points from baseline) and with haloperidol $10 \mathrm{mg} /$ day (by 22.8 points from baseline) compared to recipients of the sub-therapeutic $8 \mathrm{mg}$ /day dose of sertindole. In this study, sertindole $16 \mathrm{mg}$ /day was at least as effective as the two higher doses. The results of these two comparative trials with haloperidol have suggested that sertindole was more effective against negative symptoms; moreover, sertindole $16 \mathrm{mg} /$ day was as efficacious as $20 \mathrm{mg} /$ day, suggesting that the initial titration should be made to $16 \mathrm{mg} /$ day. They also confirm that $8 \mathrm{mg}$ /day was suboptimal with respect to efficacy. ${ }^{46,47}$ Additional evidence provided from the overmentioned studies is that sertindole $24 \mathrm{mg} /$ day did not appear to be any more efficacious than $20 \mathrm{mg}$ /day, the recommended prescribed dose range is $16-20 \mathrm{mg}$ /day. The longer term efficacy of sertindole was assessed in a double-blind, 12-month trial comparing $24 \mathrm{mg}$ /day sertindole with $10 \mathrm{mg}$ /day haloperidol in 282 outpatients with schizophrenia. ${ }^{48}$ At primary endpoint, time to treatment failure was numerically superior in sertindole-treated patients compared to haloperidol-treated patients, even if this difference did not reach statistical significance. After 2 months of treatment, PANSS total score significantly decreased from baseline in sertindole group more than in haloperidol group $(P<0.05)$, although non statistically significant differences between groups were found after 12 months of treatment. Moreover, at 1-year follow-up, more haloperidol patients had left the study due to: any reason, adverse events and lack of efficacy, suggesting better compliance for the sertindole compared with the haloperidol group.

A double-blind, parallel-group, flexible-dose, multicenter study compared the efficacy and tolerability of sertindole $(12-24 \mathrm{mg} /$ day; $\mathrm{n}=98)$ with risperidone $(4-10 \mathrm{mg} /$ day; 
$\mathrm{n}=89$ ) in 187 out- and inpatients with various subtypes of schizophrenia who had to be at least moderately ill. The study terminated prematurely because of the temporary withdrawal of sertindole from the European Union market; however, although early termination reduced the power of the study, some significant between-group differences emerged. After 12 weeks of treatment, improvements in positive and negative symptoms were seen in both treatment groups. Sertindoletreated patients had a greater reduction in PANSS negative subscale scores. However, data for leaving the study early were available but the total was high (35.8\%). Adverse effects were only reported if $>5 \%$ occurrence. ${ }^{49}$

The recent observational NICHE study was a retrospective, single-arm, observational crossover study designed to evaluate complementary benefit/risk during the temporary suspension of the marketing authorizations for sertindole. The study included 344 patients who had two nonconsecutive treatment periods with sertindole. From the patients included in this study, around $17 \%$ followed four treatment periods. From the remaining patients, around $45 \%$ followed three treatment periods and around $38 \%$ followed two treatment periods. The results showed that a subset of patients switched to sertindole had an improvement in their clinical symptoms, as documented by the decrease in the number and duration of hospitalizations, as well as suicide attempts. ${ }^{50}$

Leucht et $\mathrm{al}^{51}$ have recently conducted a meta-analysis comparing nine second-generation antipsychotics with firstgeneration drugs for overall efficacy, positive, negative, and depressive symptoms, relapse, quality of life, extrapyramidal side-effects, sedation, and weight gain. Results showed that five second-generation drugs (aripiprazole, quetiapine, sertindole, ziprasidone, and zotepine) were not significantly different from first-generation antipsychotics in their effects on overall, positive, and negative symptoms, whereas clozapine, amisulpride, olanzapine, and risperidone, were more efficacious than first-generation drugs. Olanzapine (relative risk $\mathrm{RR}=0.67 ; 95 \%$ confidence interval CI: 0.49-0.92), risperidone $(\mathrm{RR}=0.74,95 \% \mathrm{CI}: 0.63-0.87)$, and sertindole $(\mathrm{RR}=0.17 ; 95 \% \mathrm{CI}: 0.04-0.73)$ proved to be significantly better than first-generation drugs on relapse prevention. With regard to quality of life, only clozapine (Hedge's $g=-0.24$; 95\% CI: $-0.46-0.01 ; P=0.039$ ), sertindole (Hedge's $\mathrm{g}=-0.44 ; 95 \% \mathrm{CI}:-0.483-0.05 ; P=0.027)$, and amisulpride (Hedge's $\mathrm{g}=-0.31 ; 95 \% \mathrm{CI}:-0.60-0.03 ; P=0.030$ ) were better than first-generation drugs. ${ }^{51}$

The cited studies documented that sertindole is as effective as haloperidol and risperidone in the treatment of positive symptoms, but it may be more effective than these agents in the treatment of negative symptoms. These results need to be confirmed by further studies, as no randomized evidence compares sertindole with other second generation antipsychotic drugs such as amisulpride, aripiprazole, clozapine, olanzapine, quetiapine, ziprasidone or zotepine, as recently suggested by Komossa et $\mathrm{al}^{52}$ whose review was based on strict inclusion criteria with sensitivity analyzes in order to exclude studies with potentially skewed data and probable sources of bias, including selective reporting, incomplete outcome data, randomization and blinding methods.

\section{Cognitive symptoms}

As previously reported in the Pharmacological Profile section, sertindole has no effect on muscarinic or histaminic $\mathrm{H} 1$ receptors, and, as a result, is not associated with anticholinergic side effects or sedation..$^{53}$ A 12-week, randomized double-blind study has compared the effect of sertindole (10 to $24 \mathrm{mg} /$ day) and haloperidol on cognitive functioning in 40 patients with schizophrenia. Neurocognitive assessment included elementary cognitive processes (reaction time), working memory (digit span), and executive function (Wisconsin Card Sorting test). The results evidenced that sertindole was superior to haloperidol in most of the applied tasks after a short (4-week) as well as an extended (12-week) treatment period. The beneficial effects observed with sertindole also seemed to be specific to cognitive independently of motor function. ${ }^{54}$ In this trial, however, a potential bias cannot be excluded, as a number of studies reported that first-generation antipsychotics, like haloperidol, were not effective in cognitive impairment, ${ }^{55}$ and they could even worsen cognitive functions. ${ }^{56,57}$ Moreover, further caution in the interpretation of the results obtained by Gallhofer et $\mathrm{al}^{54}$ is needed, as the haloperidol group was also receiving anticholinergic medication.

Another proposed mechanism underlying cognitive dysfunction and negative symptoms in schizophrenia is dopaminergic hypofunction in the prefrontal cortex (PFC).$^{58}$ Sertindole, as well as risperidone, markedly increased the extracellular levels of DA in medial PFC and nucleus accumbens of conscious rats; differently from risperidone, sertindole also enhanced extracellular levels of acetylcoline (ACh) and glutamate (Glu) in the rat $\mathrm{mPFC} .{ }^{20}$ It has been hypothesized that potentiation of insufficient dopaminergic and glutamatergic transmission and strengthening of the cholinergic transmission in the PFC may explain the beneficial effects of sertindole on cognitive function in patients with schizophrenia, ${ }^{20,54}$ and the superior effect against negative 
symptoms compared to risperidone reported in a clinical study on sertindole and risperidone. ${ }^{49}$

A recent PET (18F)-fluorodeoxyglucose (FDG) and magnetic resonance imaging (MRI) study on fifteen patients with schizophrenia receiving sertindole up to $24 \mathrm{mg}$ /day or haloperidol up to $16 \mathrm{mg}$ /day under a blind, cross-over condition, showed that sertindole, compared to haloperidol, had an activating function in the dorsolateral and anterior prefrontal cortex. Comparison of unmedicated patients and normal controls indicated that sertindole tended to change the relative metabolic rate of frontal regions in the direction of normal values. ${ }^{59}$ However, a major limitation of trials addressing the effect of sertindole on cognitive impairment in schizophrenia is that haloperidol was the comparator drug in most of the studies; consequently, the effective role of sertindole in treating cognitive dysfunctions has been incompletely explored, and comparisons with second-generation drugs are needed.

\section{Safety and tolerability}

Sertindole safety profile has been evaluated in several clinical trials and large epidemiological studies involved in post-marketing surveillance programme. ${ }^{60-64}$ Additional information is provided in the product monograph. ${ }^{38}$

\section{Common adverse events and overdose}

Results from a safety database of approximately 2500 patients treated with sertindole in clinical trials evidenced that $89 \%$ of patients reported at least one treatment-emergent adverse event. The most commonly reported adverse events associated with sertindole treatment were headhache $(33.8 \%$ of patients), insomnia (31.3\%), rhinitis/nasal congestion $(28.5 \%)$, and, in male patients, decreased ejaculatory volume $(21.8 \%)$. Sertindole is not associated with sedation; with regard to anticholinergic effects, dry mouth and constipation occurred in $\geq 10 \%$ of patients. These adverse events (AEs) did not appear to be dose-related ${ }^{65}$ A relationship with sertindole dose was observed for orthostatic hypotension, whose incidence was higher during the titration phase and proportionally increased with increasing sertindole dose; $13 \%$ of patients treated with sertindole $24 \mathrm{mg} /$ day vs $5 \%$ of patients treated with $8 \mathrm{mg} /$ day $(P<0.05)$ experienced this AE. ${ }^{47} \mathrm{In}$ the same trial, discontinuation rates due to AEs were low ( $\leq 0.4 \%$ ); nevertheless, $3.2 \%$ of male patients chose to stop treatment because of the sexual adverse event of decreased ejaculatory volume (DEV).$^{47}$ The mechanism of a reduction in ejaculation volume during treatment with sertindole, a side-effect usually not associated with erectile disturbances or decreases in libido, is as yet not precisely known; this effect might be due to the $\alpha_{1}$ noradrenergic antagonistic properties of sertindole, and it probably does not involve retrograde ejaculation as no semen could be detected in the urine after orgasm. ${ }^{45}$ An alternative mechanism has been suggested involving calcium channels on the vas deferens as, in vitro, contractions of the vas deferens are known to be affected by calcium channel antagonists. Sertindole, such as other antipsychotics, has the property to block sodium and calcium influx channels, ${ }^{66}$ so this may contribute to DEV.

Experience with sertindole in acute overdose is limited; the drug appears to be relatively safe in overdose on its own, as patients taking estimated dosages up to $840 \mathrm{mg}$ have recovered without sequelae. A recent case-report evidenced that sertindole and fluoxetine overdose had a benign outcome; ${ }^{67}$ however, fatal cases have occurred. ${ }^{38}$ Reported signs and symptoms of overdose were dyspnea, somnolence, slurred speech, tachycardia, hypotension, transient AV-block and QT-c prolongation; cases of Torsades de Pointes (TdP) have been observed, often when sertindole was combined with other drugs known to induce TdP. In overdose cases, due to the long half-life of sertindole in humans (3-5 day), and its QT prolongation capacity, therapeutic drug monitoring would be useful to follow the sertindole concentrations. ${ }^{68}$

\section{EPS and akathisia}

Data provided by clinical trials suggest that sertindole may produce EPS comparable to placebo. As assessed by Simpson-Angus Rating Scale (SAS), Barnes Akathisia Scale (BAS), and Abnormal Involuntary Movements Scale (AIMS), there were no statistically significant differences between sertindole (8-24 $\mathrm{mg}$ /day $\mathrm{mg}$ ) and placebo for the incidence of EPS, use of medication to avoid extrapyramidal symptoms, akathisia, cogwheel rigidity, hypertonia and tremor. This was in sharp contrast to haloperidol (4-16 mg/day), which showed worsening in most of the EPS and akathisia scales. ${ }^{45-48}$ In the multicenter, 8-week, placebo-controlled trial by Zimbroff et al, ${ }^{46}$ the incidence of EPS was reported in $13.2 \%-23.6 \%$ of patients treated with sertindole (12-24 mg/day) compared with $27.4 \%$ of those given placebo, and with $43.7 \%-55.7 \%(P<0.05)$ of patients treated with haloperidol (4-16 mg/day). The long-term haloperidol-controlled trial substantiated the favorable safety profile of sertindole, as a significantly lower incidence of akathisia ( $14 \%$ vs $24 \%$ ), tremor ( $9 \%$ vs $21 \%$ ), and hypertonia ( $9 \%$ vs $18 \%$ ) was reported in patients treated with sertindole (24 mg/day) compared with those treated with haloperidol (10 mg/day). ${ }^{48}$ Lewis et al, ${ }^{69}$ in their meta-analysis of two 
haloperidol-controlled trials, ${ }^{46,48}$ noted that the incidence of movement disorders was less frequent with sertindole (24 mg/day) than with haloperidol (10 mg/day), and not significantly different from placebo. Regarding risk ratio, sertindole $24 \mathrm{mg}$ /day had a statistically significant lower risk than haloperidol $10 \mathrm{mg} /$ day of akathisia (relative risk $0.47 ; P=0.0004)$, hypertonia $(0.45 ; P=0.003)$, and tremor $(0.36 ; P=0.00009)$.

The short-term, double-blind, risperidone-controlled trial by Azorin et al, ${ }^{49}$ showed that $19 \%$ of patients treated with sertindole vs $28 \%$ of those given risperidone reported EPSrelated AEs, although this difference did not reach statistical significance.

These limited data suggest that akathisia and parkinsonism may occur less frequently with sertindole than with risperidone. However, relatively high risperidone dose ranges of 4 to $10 \mathrm{mg} /$ day (mean $6.6 \mathrm{mg} /$ day) in the over-mentioned study must be taken into account. ${ }^{52}$

Long-term treatment with antipsychotic drugs, especially at high dosages, is associated with the risk of tardive dyskinesia (TD). Regarding sertindole, data on the potential risk for TD are still lacking, because of the limited duration of clinical trials and follow-ups. However, based on the low incidence of parkinsonism and akathisia with sertindole in clinical trials, it may be hypothesized that this drug might have a low incidence of TD with long-term use. In animal models of TD, the chronic administration of sertindole or olanzapine did not produce a significant incidence of rat oral chewing movements, whereas chronic haloperidol treatment typically produces late-onset, purposeless oral chewing movements in laboratory rats with a prevalence of $60 \%$. To the extent that this rat condition can be seen to model human TD, these animal results may predict low TD liability of sertindole and olanzapine chronic use. ${ }^{70}$

A trial including 151 patients switched from conventional antipsychotics to sertindole showed that three out of four patients who had developed tardive dyskinesia, dystonia and/or akathisia, apparently recovered from the movement disorders after switching to sertindole; in one patient, sertindole monotherapy was not sufficient to reduce the movement effects, but combination treatment with tetrabenazine resulted in a greater reduction in extrapyramidal symptoms. $^{71}$

The meta-analysis by Leucht et $\mathrm{al}^{51}$ showed that all second-generation antipsychotics were associated with much fewer EPS than haloperidol; however, with the exception of clozapine, olanzapine, and risperidone, second-generation drugs, including sertindole, have not been shown to be better than low-potency first-generation antipsychotics with regard to EPS. Furthermore, amisulpride, clozapine, olanzapine, quetiapine, risperidone, sertindole and zotepine were associated with more weight gain than was haloperidol, whereas aripiprazole and ziprasidone were not.

\section{Endocrine and metabolic side effects}

In addition to EPS, important AEs associated with atypical antipsychotics are the drugs' prolactin profile, weight gain, and alteration of glucose and lipid metabolism. Sertindole does not cause clinically significant increases in prolactin, in short- or long-term clinical studies. ${ }^{45,47,48}$ Similarly to other atypical antipsychotics, sertindole appears to induce a moderate weight gain, presumably related to the high affinity for $5-\mathrm{HT}_{2 \mathrm{C}}$ receptors. In the placebo-controlled clinical trials, there was a statistically significant greater mean weight gain among participants taking sertindole $(20 \mathrm{mg}$, mean weight gain of $3.3 \mathrm{~kg}$ ) as compared to placebo (mean weight gain of $0.8 \mathrm{~kg} ; P<0.05){ }^{69}$

According to Hale et al, ${ }^{47}$ at six weeks, all of the sertindole groups $(8,16,20$ or $24 \mathrm{mg} /$ day) showed an increase in body weight from baseline to final evaluation ranging from $1.3 \mathrm{~kg}$ to $1.9 \mathrm{~kg}$, all of which represented a statistically significant different weight change than that recorded for the haloperidol treatment group $(-0.1 \mathrm{~kg})$. In the longterm trial, data on the incidence of weight gain were taken into account: at one year, a significantly greater number of sertindole treated participants $(n=19 / 141)$ were reported to have gained weight as compared to haloperidol treated participants $(n=3 / 141)$. Over one year of treatment with sertindole $24 \mathrm{mg} /$ day, a mean weight gain of $4.6 \mathrm{~kg}$ from baseline was observed. ${ }^{48}$ In the double-blind, risperidonecontrolled trial, patients in both treatment groups showed a small increase in weight (sertindole, $2.1 \pm 3.9 \mathrm{~kg}$; risperidone, $1.5 \pm 3.7 \mathrm{~kg}$ ) and body mass index (sertindole, $0.7 \pm 1.3$; risperidone, $0.5 \pm 1.2$ ) with no significant difference between the two treatments. Nevertheless, there was a significant difference for the change in weight from baseline in $\mathrm{kg}$, favoring risperidone. ${ }^{49}$

The multicenter, multinational, cohort European Postmarketing Observational Sertindole (EPOS) Study, was designed to compare the safety of treatment with sertindole vs other antipsychotics. ${ }^{64}$ During the first 4 weeks of sertindole treatment $650(62 \%)$ of the 1,050 patients remaining in the cohort experienced at least one AE; after 4 weeks, a slight increase was observed in the number of metabolic and nutritional disorder, and weight increase was observed in $6 \%$ of patients. 
Data on the effect of sertindole on glucidic and lipidic metabolism are still sparse and incomplete. Minor increases in plasma levels of glucose, cholesterol and triglycerides have been reported in short-term studies; ${ }^{65}$ increased mean plasma cholesterol levels in one trial $(P<0.05$ vs placebo $)$ were documented. ${ }^{45}$

\section{Cardiovascular side effects}

The propensity of sertindole to prolong the QT interval is well recognized. The QT interval is an ECG measure that consists of both depolarization and repolarization, beginning with the onset of ventricular depolarization ( $Q$ wave) and ending with completion of repolarization (T wave). In clinical practice, its corrected form for heart rate (QTc) is used, since the QT interval shortens with increasing heart rates. Prolonged QTc interval increases the risk of cardiac arrhythmias and may predispose to the development of ventricular tachyarrhythmias such as TdP and ventricular fibrillation. ${ }^{72} \mathrm{TdP}$ is a malignant ventricular arrhythmia that is associated with long QT intervals and is generally unresponsive to the usual antiarrhythmic drugs; it can progress to ventricular fibrillation and sudden death. QTc intervals are usually around $400 \mathrm{msec}$ in duration, and values below 440 are considered normal. The greater the duration, the more likely torsade de pointes becomes, but $500 \mathrm{msec}$ has frequently been used as a cut-off because longer QTc interval measures are associated with substantially higher risk.

Sertindole showed a dose-dependent increase in the QTc interval that averaged $22 \mathrm{msec}$ at usual therapeutic doses, whereas a QTc interval $<500 \mathrm{msec}$ was observed in approximately $2 \%$ of 2194 sertindole-treated patients. ${ }^{73,74}$ In a multicenter, double-blind, placebo-controlled study by Zimbroff et al, ${ }^{46}$ mean QTc changes for the sertindole 12-, 20-, and 24-mg groups and placebo were 7/11 msec, 17/19 msec, and 19/21 msec, and -5/-4 msec, respectively; however, no ECG changes could be related to clinical signs or symptoms (eg, syncope), and no arrhythmias (ie, TdP) were seen in any of the sertindole treatment groups. Moreover, QTe value of $\geq 500$ was observed in $1 \%$ of the patients in the sertindole groups, whereas no patient in the placebo group had a QT or QTc interval that exceeded $500 \mathrm{msec}$ at any point during the study.

In a long-term study ${ }^{48}$ only one participant from the sertindole group ( $24 \mathrm{mg}$ ) had a QT interval that exceeded $500 \mathrm{msec}$. However, 11 sertindole treated participants had QTc intervals of at least $500 \mathrm{msec}$, compared to none in the haloperidol treated group ( $8 \%$ vs $0 \%, P<0.05)$. In the risperidone-controlled trial by Azorin et al, ${ }^{49}$ significantly more sertindole-treated patients reported QT prolongation ( $22.7 \%$ versus $4.5 \%)$; moreover, of the 12 (12\%) premature drop-outs during sertindole treatment, six were due to QTc interval prolongation. However, although a close relationship between drug concentration and the extent of QT prolongation can often be observed, this does not mean that such a relationship is also present with respect to the risk for TdP; an electrophysiologic study of sertindole in a rabbit heart model of TdP showed that, despite the QT prolongation, the torsadogenic potential of sertindole seems to be remarkably low. ${ }^{14}$

Beyond sertindole, many conventional and atypical antipsychotics have been related to prolongation of QTc. ${ }^{75}$ The most compelling evidence exists with thioridazine which has been reported to increase QTc about of $30 \mathrm{msec}$, followed by ziprasidone $(15.9 \mathrm{msec})$, haloperidol $(7.1 \mathrm{msec})$, quetiapine $(5.7 \mathrm{msec})$, risperidone $(3.6-3.9 \mathrm{msec})$, and olanzapine $(1.7 \mathrm{msec}) .{ }^{76} \mathrm{It}$ is noteworthy that not all drugs that prolong the QTc interval produce TdP and sudden death; all drugs associated with fatal arrythmias and sudden death have in common the fact that they affect myocardial repolarization blocking the delayed rectifier potassium $\mathrm{IKr},{ }^{77,78}$ a channel expressed by the HERG gene. ${ }^{72}$ The low myocardium/plasma ratio, and the limited distribution to the myocardium of sertindole, may limit its effective concentration at Ikr sites. ${ }^{75}$

Generally, drug-induced arrhythmias are more likely to occur in patients with pre-existing QT prolongation, which may be congenital or due to many causes (myocardial disease, left ventricular hypertrophy, malnourishment, alcoholism, ischemia, or potassium depletion). Moreover, drug interactions must be taken into account, as drugs with minimal QTc interval prolongation may interact with other compounds, competing for the same metabolic way. ${ }^{79}$ Recently, the role of QT interval as the only marker for estimation of a drug's proarrhythmic risk has been questioned, as for QTc durations $<500 \mathrm{~ms}$, the QTc interval lacks a clear correlation with TdP risk; conversely, T-wave morphology changes (notches, asymmetry, and flatness) are associated with inhibition of the IKr channel and might be more important than the QTc interval in predicting TdP occurrence. Prominent T-wave morphology changes occurred during sertindole treatment and in some cases without concomitant prolongation of the QTc interval, have been found in an ECG study on 37 schizophrenic patients who were taking sertindole $16 \mathrm{mg} .{ }^{80}$ Further ECG results from the same sample have recently been published..$^{81}$ Beyond T-wave morphology and QTc interval, other markers, including QTc dispersion and Tpeak-Tend, may better correlate with cardiac arrhythmia risk. QT disper- 
sion (QTD; normal range $=40-60 \mathrm{~ms}$, with QTD $>100 \mathrm{~ms}$ ) is the interlead difference between the shortest and longest QTc interval on a 12-lead surface ECG, whereas Tpeak-Tend, whose normal range is $80-100 \mathrm{~ms}$, consists in the time from the peak of the T-wave to the end of the T-wave. Results from the ECG study on 37 patients switched to sertindole showed that the increase in QTc interval (mean prolongation: $20 \pm 23 \mathrm{~ms}$ ) induced by sertindole, was not associated with a proportional increase in QTD. TPTE, however, increased from 81 to $88 \mathrm{~ms}(P=0.05)$, values still considered within the normal range of TPTE in healthy subjects and, probably, of limited clinical relevance. ${ }^{81}$

Until a wide experience in estimating the total cardiac safety of psychiatric drugs is reached, clinicians should be aware of cardiovascular side effects, especially for the patients at risk, such as elderly or subjects with pre-existing cardiovascular disease.

\section{Suicide and mortality rates}

Several postmarketing epidemiological studies have been conducted to better define sertindole safety profile and to identify all deaths during sertindole treatment and the causes thereof. ${ }^{50,60-64}$ The multicenter, multinational, cohort EPOS Study, ${ }^{64}$ aimed to compare the safety of treatment with sertindole vs other antipsychotics under normal clinical practice conditions, was designed to include a total of 12,000 patients recruited in 13 European countries, and to follow patients for at least one year of therapy. As a result of the temporary removal of sertindole from the market, the study was prematurely terminated on December 1998, less than 18 months after it had begun recruitment. A total of 2,321 patients were enrolled (1,064 treated with sertindole and 1,257 treated with other antipsychotics) at 226 centers in ten European countries. As the study was prematurely terminated, only 140 patients (64 taking sertindole and 76 taking other antipsychotics) completed the full observation period. The overall mortality rates were 1.45 deaths per 100 patient-years of exposure (PYE) (95\% CI: 0.53-3.16) in the

Table 2 Mortality rates from clinical trials and European Safety and Exposure Survey (ESES)

\begin{tabular}{lll}
\hline & Clinical trials & ESES \\
\hline Patients $(\mathrm{n})$ & $303 \mathrm{I}$ & 8608 \\
Deaths $(\mathrm{n})$ & 29 & 35 \\
All-cause & $\mathrm{I} .52(0.96-2.13)$ & $0.92(0.64-\mathrm{I} .35)$ \\
mortality/ I00 PYE $(\mathrm{Cl}) *$ & & \\
Cardiac mortality/ I00 PYE $(\mathrm{Cl}) *$ & $0.3 \mathrm{I}(0.1 \mathrm{I}-0.688)$ & $0.35(0.17-0.65)$ \\
\hline$* \mathrm{Cl}=95 \%$ Confidence Intervals. & &
\end{tabular}

sertindole group $(\mathrm{n}=1053)$ and 1.50 deaths per 100 PYE (95\% CI: $0.72-2.76)$ in the nonsertindole group $(n=1257)$. The deaths were categorized by cause into noncardiac, cardiac, uncertain cause, and suicide. Suicide was the most common cause of death in the sertindole group, with three completed suicides occurring from start of treatment up to five days after discontinuation; moreover, in the sertindole group, no fatal cardiac AEs occurred within the study period or 5 days of cessation of sertindole treatment. Noncardiac death was the most common cause of death in the nonsertindole group.

The European sertindole safety and exposure survey (ESES), a follow-up study of 8608 patients treated with sertindole in six European countries, showed an overall mortality rate of 0.92 deaths per 100 PYE (95\% CI: 0.64-1.35), with no excess cardiovascular deaths ( 0.35 deaths per 100 PYE; $95 \%$ CI: $0.17-0.65)$ and suicide (0.21 deaths per 100 PYE; 95\% CI: 0.09-0.41). Serious nonfatal adverse events included QTc interval prolongation, arrhythmias, convulsions and suicide attempts (see Table 2). A nested case-control study included in this survey identified age, cardiovascular and metabolic disorders as risk factors for cardiac/sudden death in patients receiving sertindole. ${ }^{60}$

The sertindole safety survey (SSS) was a multicenter retrospective survey of 1,432 patients treated with sertindole in 11 European contries after its market suspension; the overall mortality rate was 0.51 deaths per $100 \mathrm{PYE} \mathrm{(95 \%} \mathrm{CI:}$ 0.23-0.97). When considering only exposure after market suspension of sertindole (1,396 PYE), the mortality rate was 0.64 deaths per 100 PYE (95\% CI: 0.30-1.22). Suicide rates were 0.11 deaths per 100 PYE (95\% CI: 0.01-0.41) and 0.14 deaths per PYE (95\% CI: 0.02-0.52) after market removal of sertindole; sudden death rates were 0.17 deaths PYE (95\% CI: 0.04-0.50) before, and 0.21 deaths PYE (95\% CI: 0.04-0.63) after market suspension of sertindole, respectively. Among non-fatal SAEs (4.95 per 100 PYE; 95\% CI: 3.96-6.10) 15 cases (0.85 per 100 PYE; 95\% CI: $0.48-1.41)$ of QT prolongation with no clinical effects were reported. ${ }^{62}$

The Sertindole Cohort Prospective (SCoP) study was a prospective, randomized, partially blinded, active-controlled, multinational trial, major prospective post- marketing surveillance study aimed to compare the safety of sertindole with that of risperidone under normal conditions of use in nearly 10,000 schizophrenic patients. The first primary endpoint was all-cause mortality, and the second was hospitalization with arrhythmia; secondary endpoints included cause-specific fatal events, suicide attempts, hospitalizations, and treatment duration. After taking either sertindole $(n=4,930)$ or 
risperidone $(n=4,928)$ for one year, there were 64 deaths in the sertindole group and 61 deaths in the risperidone group; this difference in all-cause mortality was not statistically significant. However, sertindole was associated with a fivefold greater risk of sudden cardiac death than risperidone $(\mathrm{HR}=5.1,95 \% \mathrm{CI}: 1.5-17.9){ }^{61,63}$

A retrospective cohort study recently evaluated the adjusted incidence of sudden cardiac death among current users of antipsychotic drugs in 44,218 and 46,089 baseline users of single typical and atypical drugs, respectively, and 186,600 matched nonusers of antipsychotic drugs. The study did not include sertindole, as it has not yet been marketed in the United States. Results obtained showed that current users of typical and of atypical antipsychotic drugs had higher rates of sudden cardiac death than did nonusers of antipsychotic drugs, with adjusted incidence rate ratios of 1.99 (95\% CI: 1.68-2.34) and 2.26 (95\% CI: 1.88-2.72), respectively. On the other hand, former users of antipsychotic drugs had no significantly increased risk (incidence rate-ratio, 1.13; 95\% CI: 0.98-1.30). For both conventional and atypical antipsychotics, the risk of sudden cardiac death increased with an increasing dose; moreover, there was a dose-response trend for each of the six frequently prescribed drugs (haloperidol, thioridazine, clozapine, olanzapine, quetiapine, and risperidone), a trend that was significant in the case of thioridazine $(P=0.005)$ and of borderline significance in the case of risperidone $(P=0.05) .{ }^{82}$

Tiihonen et $\mathrm{al}^{83}$ have recently concluded a populationbased cohort study (FIN11 study) consisting of an 11-year follow-up of mortality in 66881 Finnish patients with schizophrenia treated with first- and second-generation antipsychotics. The study did not include separate analysis for sertindole, as altogether aripiprazole, sertindole, and ziprasidone accounted for $0 \%-3 \%$ of all defined daily doses (DDDs) (0.45 million of 156.46 million). Results showed that the overall risk of death was lower $(\mathrm{HR}=0.68 ; 95 \% \mathrm{CI}$ : $0.65-0.71 ; P<0.0001)$ during the current use of any antipsychotic drug than it was with no antipsychotic use. The risk of death was significantly lower in patients with long-term (7-11 years) antipsychotic treatment than in those who had not used any antipsychotic drugs during follow-up (HR $=0.81 ; 95 \%$ CI: 0.77-0.84; $P<0.0001)$. Furthermore, no signs of increased risk of death from ischemic heart disease were noted after 7-11 years of cumulative exposure to antipsychotic treatments. Comparisons of specific antipsychotics versus perphenazine showed that current use of quetiapine, haloperidol, and risperidone were associated with an increased mortality $(41 \%, 37 \%$, and $34 \%$, respectively) and clozapine with a reduced mortality (-26\%), suggesting that the increase in the use of second-generation antipsychotics (from 13\% to 64\% during 1996-2006), rather than first-generation drugs, did not have a harmful effect on lifeexpectancy of patients with schizophrenia, with the possible exception of quetiapine and risperidone.

\section{Conclusion}

Sertindole is a second-generation antipsychotic with a peculiar pharmacological and clinical profile. It has recently been reintroduced in the market in several European countries for the treatment of schizophrenia under special safety conditions (ie, no first choice treatment, not for emergency treatment, ECG observation).

Like other second-generation antipsychotics, sertindole has a high affinity for serotonin $5-\mathrm{HT}_{2 \mathrm{~A}}$ and $5-\mathrm{HT}_{2 \mathrm{C}}$ and $\alpha_{1}$ adrenergic receptors; its affinity for dopamine $\mathrm{D}_{2}$ receptors is more pronounced in limbic rather than nigrostriatal system. Due to its pharmacological characteristics, sertindole is expected to exert antipsychotic activity with limited risk of EPS. Moreover, sertindole showed modest affinity for $\mathrm{H} 1$-histaminergic and muscarinic receptors, thus suggesting a low potential to cause sedation or cognitive impairment Sertindole is metabolized in the liver by the cytochrome P450 isoenzymes CYP2D6 and CYP3A4 and has a plasma halflife of approximately three days. The relatively long half-life in comparison to other atypical antipsychotics allows once daily administration and, theoretically, may result in low fluctuations in plasma and target receptor concentrations of the drug at steady-state. ${ }^{84}$

After a comprehensive clinical development programme leading to the introduction of sertindole in the EU in 1996, its temporary withdrawal from the market due to cardiovascular safety concerns, led to the need to examine potential cardiovascular toxicity more than clinical efficacy; consequently, data on clinical efficacy are still limited. Although sertindole appeared to be generally well tolerated, more effective than placebo and at least as effective as haloperidol and risperidone on positive symptoms, the evidence is therefore very incomplete as studies were short-term and that long-term effectiveness studies are scarcely available, clearly limiting external validity.

Except for risperidone, no randomized trials have compared sertindole with other second-generation antipsychotic drugs. Further "head-to-head" comparisons with other second-generation antipsychotic drugs are strongly needed. Preliminary data from pre-clinical, animal model and clinical studies suggest that sertindole should have a beneficial effect on schizophrenia-related cognitive disturbances. 
In clinical practice, sertindole can be administered orally once daily at the recommended starting dose of $4 \mathrm{mg} /$ day, which can be titrated by increments of $4 \mathrm{mg}$ after $4-5$ days on each dose until the optimal daily maintenance dose, within the range of 12-20 mg, has been achieved. Doses should be halved for concomitant administration with strong CYP2D6 and CYP3A4 inhibitors. Renal impairment does not significantly change sertindole pharmacokinetics, reflecting its extensive hepatic metabolism. Thus, in patients with mild, moderate, and severe renal impairment, dosage adjustment is not necessary, while dose reduction is required in hepatically impaired patients, as the clearance of sertindole decreases in this population.

Information on the efficacy and safety of sertindole in special populations such as children and adolescents, elderly patients, and subjects with treatment-refractory schizophrenia is still limited or lacking.

Nevertheless, taking into account these weaknesses, the only available data demonstrated noninferiority to risperidone; sertindole may induce fewer movement disorders than risperidone - at least when the latter is given in relatively high doses (between 4 and $12 \mathrm{mg} /$ day). However, sertindole may be associated with more QTc prolongation, weight change, and male sexual dysfunction. Moreover, data are limited on the effectiveness of sertindole in the maintenance treatment of schizophrenia.

With regard to the main tolerability and safety issues of sertindole, its effect on the QT interval is of concern. Like many antipsychotics, both conventional and atypical, sertindole can prolong the QTc interval. The length of the QTc interval has been associated with the risk of sudden death after myocardial infarction; although it is not proven that drug-induced QTc interval prolongation behaves in the same manner, it is thought to be a predictor for TdP episodes and is considered a risk factor for sudden cardiac death. Among atypical antipsychotics, sertindole, iloperidone, ${ }^{85}$ and ziprasidone, (which was not licensed in the UK because of concerns about potential cardiotoxicity), are the compounds associated with a greater risk of QTc prolongation. Among conventional antipsychotics, the most compelling evidence exists with thioridazine, that prolongs the QT interval, blocks the $\mathrm{IKr}$ channel, and is associated with numerous cases of TdP and an excess number of sudden death. Thioridazine prolongs the QTc interval about $30 \mathrm{msec}$, whereas the maximum prolongation of QTc interval with sertindole is 20-22 msec (depending on dose). However, although QTc prolongation is a risk factor for serious arrythmias, there is considerable evidence in the literature that the relation between QTc prolongation and development of fatal arrythmias is not so direct. The low myocardium/plasma ratio, and the limited distribution to the myocardium of sertindole, may limit its arrhythmogenic potential. Furthermore, cardiovascular risk is in fact associated with the risk of drug interaction and with the characteristics of patients exposed to the treatment in clinical practice. Patients with schizophrenia are considered a population at higher risk for sudden death independent of drug treatment. Before prescribing sertindole, risk factors for drug-induced tachyarrythmias including female sex, cardiac hypertrophy, heart failure, bradycardia, electrolyte imbalances (mainly hypokalemia), congenital QT syndrome and drug interactions must be taken into account. The administration of sertindole, as recommended for other antipsychotics, is contraindicated if a QTC interval of more than $450 \mathrm{msec}$ in males or $470 \mathrm{msec}$ in females is observed at baseline. In order to substantially reduce cardiovascular risk, ECG monitoring is required before and during treatment with sertindole. ECG monitoring should be carried out at baseline, upon reaching steady state after approximately three weeks, or when a dosage of $16 \mathrm{mg}$ is reached, and again after three months of treatment. Furthermore, it is recommended to conduct additional ECG monitoring prior to and following any dosage change of sertindole, or of concomitant medication, which may increase sertindole concentration.

The potential for cardiac adverse events associated with sertindole is balanced by a tolerability profile characterized by the low liability to induce sedation, hyperprolactinemia, and EPS. With regard to tardive dyskinesia, there are no available data. During treatment with sertindole, a moderate weight gain has been documented, although most of the studies evaluated the short-term period. Common side effects observed during sertindole treatment are headache, insomnia, rhinitis/nasal congestion and, in males, sexual dysfunctions, in terms of decreased ejaculatory volume. This latter effect may have implications for overall acceptability of treatment in male patients. Preliminary evidence suggests that sertindole is not associated with a increased risk of diabetes; however, its effect on serum glucose and lipid levels need further investigation.

It can be presumed that, as coadministration of psychotropic drugs is relatively common in psychiatric clinical practice, sertindole will be reasonably prescribed in combination with other medications. Beyond the risk of synergistic effects with other drugs inducing QTc prolongation, whose use is contraindicated, the metabolic drug interaction profile of sertindole is far from being elucidated. Further studies are 
necessary to define the mechanisms, severity, and duration of the interaction with those psychotropic drugs which are more likely to be co-administered, such as other antipsychotics, antidepressants, and mood stabilizers. A better understanding of several pharmacological mechanisms (induction, competitive inhibition, or protein binding displacement) may elucidate whether there are any circumstances in which this interaction may have some clinical relevance.

At the present, it is difficult to define the place of sertindole in the management of schizophrenia in the next few years. The prolonged marketing suspension of sertindole involved considerable medical and administrative restrictions on its supply and use, and had a significant impact on research. As a consequence, available data on its efficacy, safety, and tolerability profile are still sparse and incomplete. At this time, due to cardiovascular safety concerns, sertindole is available as a second-line choice for patients intolerant to at least one other antipsychotic agent. Moreover, considering its nonsedating properties, sertindole should not be used in emergency situations for urgent relief of symptoms in acutely disturbed patients. Based on available evidence from the literature, sertindole may reduce rates of relapse and risk of re-hospitalization and, furthermore, it may have a favorable effect on quality of life.

Second-generation antipsychotic drugs differ in many properties, including efficacy, side effects, and pharmacology. Within this context, sertindole appears to have an equivalent or slightly favorable profile compared to other antipsychotic drugs when taking into account relapse rate, quality of life, and the all cause mortality rate. Nevertheless, the total amount of available information has not substantially changed in the recent years. ${ }^{84}$ Further independent and appropriately powered clinical studies, mainly direct "head-to-head" comparisons with other second-generation antipsychotic agents, are needed to clinically define the role of sertindole in the treatment of schizophrenia.

\section{Disclosure}

The authors report no conflicts of interest in this work

\section{References}

1. Saha S, Chant D, Welham J, McGrath J. A systematic review of the prevalence of schizophrenia. PLoS Med. 2005;2:e141.

2. Tamminga CA, Holcomb HH. Phenotype of schizophrenia: a review and formulation. Mol Psychiatry. 2005;10:27-39.

3. Arnt J, Skarsfeldt T. Do novel antipsychotics have similar pharmachological characteristics? A review of the evidence. Neuropsychopharmachology. 1998;18:63-101.
4. American Psychiatric Association. Practice guidelines for the treatment of patients with schizophrenia. 2nd Edition. Am J Psychiatry. 2004;16: $1-114$.

5. Gaebel W, Falkai P, Weinmann S, Wobrock T. Treatment guidelines for schizophrenia [Behandlungsleitlinie Schizophrenie]. Stuttgart, Germany: Steinkopf; 2006.

6. Miyamoto S, Duncan GE, Marx CE, Lieberman JA. Treatments for schizophrenia: a critical review of pharmacology and mechanisms of action of antipsychotic drugs. Mol Psychiatry. 2005;10:79-104.

7. Haddad PM, Sharma SG. Adverse effects of atypical antipsychotics: differential risk and clinical implications. CNS Drugs. 2007;21: 911-936.

8. Henderson DC, Cagliero E, Copeland PM, et al. Glucose metabolism in patients with schizophrenia treated with atypical antipsychotic agents: a frequently sampled intravenous glucose tolerance test and minimal model analysis. Arch Gen Psychiatry. 2005;62:19-28.

9. Luft B, Taylor D. A review of atypical antipsychotic drugs versus conventional medication in schizophrenia. Expert Opin Pharmacother. 2006;7:1739-1748.

10. Lieberman JA, Stroup TS, McEvoy JP, et al. Effectiveness of antipsychotic drugs in patients with chronic schizophrenia. New Engl J Med. 2005;353:1209-1223.

11. Stroup TS, Lieberman JA, McEvoy JP, et al. Results of phase 3 of the CATIE schizophrenia trial. Schizophr Res. 2009;107:1-12.

12. Eckardt L, Haverkamp W, Mertens M, et al. Drug-related torsade de pointes in the isolated rabbit heart: comparison of clofilium, d,1-sotalol and erythromycin. J Cardiovasc Pharmacol. 1998;32:425-434.

13. Hondeghem LM, Carlsson L, Duker G. Instability and triangulation of the action potential predict serious proarrhythmia, but action potential duration prolongation is antiarrhythmic. Circulation. 2001;103: 2004-2013.

14. Eckardt L, Breithardt G, Haverkamp W. Electrophysiologic characterization of the antipsychotic drug sertindole in a rabbit heart model of torsades de pointes: low torsadogenic potential despite QT prolongation. J Pharmacol Exp Ther. 2002;300:64-71.

15. Wilton LV, Heeley EL, Pickering RM, Shakir SA. Comparative study of mortality rates and cardiac dysrhythmias in postmarketing surveillance studies of sertindole and two other atypical antipsychotic drugs, risperidone and olanzapine. J Psychopharmacol. 2001;15:120-126.

16. Moore N. Higher cardiovascular mortality with sertindole in ADROIT: a signal not confirmed. Int J Psychiatry Clin Pract. 2002;6 Suppl 1: S3-S9.

17. Lindstrom E, Levander S. Sertindole: efficacy and safety in schizophrenia. Exp Opin Drug Saf. 2006;1825-1834.

18. Sanchez C, Arnt J, Dragsted N, et al. Neurochemical and in vivo pharmacological profile of sertindole, a limbic-selective neuroleptic compound. Drug Dev Res. 1991;22:239-242.

19. Hyttel L, Nielsen JB, Nowak G. The acute effect of sertindole on brain 5-HT2, D2 and alpha one receptors. J Neural Transm. 1992;89: $61-69$.

20. Mørk A, Witten LM, Arnt J. Effect of sertindole on extracellular dopamine, acetylcholine, and glutamate in the medial prefrontal cortex of conscious rats: a comparison with risperidone and exploration of mechanisms involved. Psychopharmacology. 2009;206:39-49.

21. Gleason S, Shannon H. Blockade of phencyclidine-induced hyperlocomotion by olanzapine, clozapine and serotonin receptor subtype selective antagonists in mice. Psychopharmacology (Berl). 1997;129: 79-84.

22. Horacek J, Bubenikowa-Valesova V, Kopecek M, et al. Mechanism of action of atypical antipsychotic drugs and the neurobiology of schizophrenia. CNS Drugs. 2006;20:389-409.

23. Wood MD, Scott C, Clarke K, et al. Pharmacological profile of antipsychotics at monoamine receptors: atypicality beyond 5-HT2A receptor blockade. CNS Neurol Disord Drug Targets. 2006;5:445-452.

24. Petit-Taboué MC, Landeau B, Barré L, et al. Parametric PET imaging of 5-HT2A receptor distribution with $18 \mathrm{~F}$-setoperone in the normal human cortex. J Nucl Med. 1999;40:25-32. 
25. Skarsfeldt T. Electrophysiological profile of the new atypical neuroleptic, sertindole, on midbrain dopamine neurons in rats: acute and repeated treatment. Synapse. 1992;10:25-32.

26. Zimbroff DL, Kane JM, Tamminga CA, et al. Controlled, dose-response study of sertindole and haloperidol in the treatment of schizophrenia. Am J Psychiatry. 1997;154:782-791.

27. Hietala J, Kuoppamaki M, Majasuo H, et al. Sertindole is a serotonin 5-HT2C inverse agonist and decreases agonist but not antagonist binding to 5-HT2C receptors after chronic treatment. Psychopharmacology. 2001;157:180-187.

28. Nyberg S, Olsson H, Nilsson U, et al. Low striatal and extra-striatal D2 receptor occupancy during treatment with the atypical antipsychotic sertindole. Psychopharmachology. 2002;162:37-41.

29. Didriksen M, Skarsfeldt T, Arnt J. Reversal of PCP-induced learning and memory deficits in the Morris' water maze by sertindole and other antipsychotics. Psychopharmacology (Berl). 2007;193:225-233.

30. Goetghebeur P, Dias R. Comparison of haloperidol, risperidone, sertindole, and modafinil to reverse an attentional set-shifting impairment following subchronic PCP administration in the rat-a back translational study. Psychopharmacology (Berl). 2009;202:287-293.

31. Rodefer JS, Nguyen TN, Karlsson JJ, Arnt J. Reversal of subchronic PCP-induced deficits in attentional set shifting in rats by sertindole and a 5-HT(6) receptor antagonist: comparison among antipsychotics. Neuropsychopharmacology. 2008;33:2657-2666.

32. Broberg BV, Glenthøj BY, Dias R, Larsen DB, Olsen CK. Reversal of cognitive deficits by an ampakine (CX516) and sertindole in two animal models of schizophrenia - sub-chronic and early postnatal PCP treatment in attentional set-shifting. Psychopharmacology (Berl). 2009;206: 631-640.

33. Idris N, Neill J, Grayson B, et al. Sertindole improves sub-chronic PCP-induced reversal learning and episodic memory deficits in rodents: involvement of 5-HT(6) and 5-HT (2A) receptor mechanisms. Psychopharmacology (Berl). 2010;208:23-36.

34. Wong SL, Linen P, Mack RJ, Granneman GR. Effects of food, antacid, and dosage form on the pharmacokinetics and relative bioavailability of sertindole in healthy volunteers. Biopharm Drug Dispos. 1997; 18:533-541.

35. Wong SL, Cao G, Mack RJ, Granneman GR. Pharmacokinetics of sertindole in healthy young and elderly male and female subjects. Clin Pharmacol Ther. 1997;62:157-164.

36. Wong SL, Menacherry S, Mulford D, et al. Parmacokinetics of sertindole and dehydrosertindole in volunteers with normal or impaired renal function. Eur J Clin Pharmacol. 1997;52:223-227.

37. Wong SL, Granneman R. Modeling of sertindole pharmacokinetic disposition in healthy volunteers in short-term dose-escalation studies. J Pharm Sci. 1998;87:1629-1631.

38. H. Lundbeck A/S. Serdolect (sertindole) monograph. Copenhagen: H. Lundbeck A/S 2005 Sep.

39. Doran A, Obach RS, Smith BJ, et al. The impact of P-glycoprotein on the disposition of drugs targeted for indications of the central nervous system: evaluation using the MDR1A/1B knockout mouse model. Drug Metab Dispos. 2005;33:165-174.

40. Wang JS, Zhu HJ, Markowitz JS, Donovan JL, De Vane CL. Evaluation of antipsychotic drugs as inhibitors of multidrug resistance transporter P-glycoprotein. Psychopharmacology. 2006;187:415-423.

41. Wong SL, Cao G, Mack RJ, Granneman GR. The effect of erythromycin on the CYP3A component of sertindole clearance in healthy volunteers. J Clin Pharmacol. 1997;37:1056-1061.

42. Haduch A, Ogórca T, Boksa J, Daniel WA. Interactions between neuroleptics and CYP2C6 in rat liver - in vitro and ex vivo study. Pharmacol Rep. 2005;57:872-877.

43. Wong SL, Locke C, Staser J, Granneman GR. Lack of multiple dosing effect of sertindole on the pharmacokinetics of alprazolam in healthy volunteers. Psychopharmacology. 1998;135:236-241.

44. Wong SL, Cao G, Mack R, Granneman GR. Lack of CYP3A inhibition effects of sertindole on terfenadine in healthy volunteers. Int $J$ Clin Pharmacol Ther. 1998;36:146-151.
45. van Kammen DP, McEvoy JP, Targum SD, et al. A randomized, controlled, dose-ranging trial of sertindole in patients with schizophrenia. Psychopharmacology. 1996;124:168-175.

46. Zimbroff A, Kane JM, Tamminga CA, et al. Controlled, dose-response study of sertindole and haloperidol in the treatment of schizophrenia: results of a phase III trial. Int J Psychiatry Clin Pract. 2000;4:55-62.

47. Hale A, Azorin JM, Kasper S, et al. Sertindole improves both the positive and negative symptoms of schizophrenia: results of a phase III trial. Int J Psychiatry Clin Pract. 2000;4:55-62.

48. Daniel DG, Wozniak P, Mack RJ, et al. Long-term efficacy and safety comparison of sertindole and haloperidol in the treatment of schizophrenia. Psychopharmacol Bull. 1998;34:61-69.

49. Azorin JM, Strub N, Loft H. A double-blind, controlled study of sertindole versus risperidone in the treatment of moderate-to-severe schizophrenia. Int Clin Psychopharmacol. 2006;21:49-56.

50. Azorin JM, Murteira S, Hansen K, ToumiM. Evaluation of patients on sertindole treatment after failure of other antipsychotics: A retrospective analysis. BMC Psychiatry. 2008;8-16.

51. Leucht S, Corves C, Arbter D, Engel RR, Li C, Davis JM. Secondgeneration versus first-generation antipsychotic drugs for schizophrenia: a meta-analysis. Lancet. 2009;373:31-41

52. Komossa K, Rummel-Kluge C, Hunger H, et al. Sertindole versus other atypical antipsychotics for schizophrenia (Review). Cochrane Database Syst Rev. 2010;2:CD006752.

53. Kasper S. Do we need another atypical antipsychotic? Eur Neuropsychopharmacol. 2008;18:S146-S152.

54. Gallhofer B, Jaanson P, Mittoux A, Tanghøj P, Lis S, Krieger S. Course of recovery of cognitive impairment in patients with schizophrenia: A randomised double-blind study comparing sertindole and haloperidol. Pharmacopsychiatry. 2007;40:275-286.

55. Mortimer AM. Cognitive function in schizophrenia-do neuroleptics make a difference? Pharmacol Biochem Behav. 1997;56:789-795.

56. Cleghorn JM, Kaplan RD, Szechtman B, Szechtman H, Brown GM. Neuroleptic drug effects on cognitive function in schizophrenia. Schizophr Res. 1990;3:211-219.

57. Cutmore TR, Beninger RJ. Do neuroleptics impair learning in schizophrenic patients? Schizophr Res. 1990;3:173-186.

58. Stone JM, Morrison PD, Pilowsky LS. Glutamate and dopamine dysregulation in schizophrenia - a synthesis and selective review. J Psychopharmacol. 2007;21:440-452.

59. Buchsbaum MS, Haznedar M, Newmark RE, et al. FDG-PET and MRI imaging of the effects of sertindole and haloperidol in the prefrontal lobe in schizophrenia. Schizophr Res. 2009;114:161-171.

60. Peuskens J, Moore N, Azorin JM, Toumi M, Cochran J. The European sertindole safety and exposure survey: a follow-up study of 8600 patients. Pharmacoepidemiol Drug Saf. 2007;16:804-811.

61. Peuskens J, Tanghøj P, Mittoux A. The Sertindole Cohort Prospective (SCoP) study: rationale, design and methodology. Pharmacoepidemiol Drug Saf. 2008;17:425-433

62. Lançon C, Toumi M, Sapin C, Hansen K. The Sertindole Safety Survey: A retrospective analysis under a named patient use programme in Europe. BMC Psychiatry. 2008;8:57.

63. Thomas SHL, Drici MD, Hall GC, et al. Safety of sertindole versus risperidone in schizophrenia: principal results of the sertindole cohort prospective study (SCoP). Acta Psychiatr Scand. 2010:1-11.

64. Kasper S, Möller HJ, Hale A. The European Post-marketing Observational Sertindole Study: an investigation of the safety of antipsychotic drug treatment. Eur Arch Psychiatry Clin Neurosci. 2010;260: 59-68.

65. Perquin LN, Steinert T. A review of the efficacy, tolerability and safety of sertindole in clinical trials. CNS Drugs. 2004;18 Suppl 2: 19-30.

66. Haverkamp W, Eckhardt L, Matz J, Frederiksen K. Sertindole: Cardiac electrophysiological profile. Int J Psychiatry Clin Pract. 2002;6: $11-20$.

67. Neckelmann D, Mæhlum E, Waage IM. Sertindole and fluoxetine overdose with benign outcome. Nord J Psychiatry. 2010 Feb 18. 
68. Canal-Raffin M, Déridet E, Titier K, Frakra E, Molimard M, Moore N. Simplified ultraviolet liquid chromatographic method for determination of sertindole, dehydrosertindole and norsertindole in human plasma. J Chromatography B. 2005;814:61-67.

69. Lewis R, Bagnall AM, Leitner M. Sertindole for schizophrenia. Cochrane Database Syst Rev. 2005;3:CD001715.

70. Gao XM, Sakai K, Tamminga CA. Chronic olanzapine or sertindole treatment results in reduced oral chewing movements in rats compared to haloperidol. Neuropsychopharmacology. 1998;19(5): 428-433.

71. Perquin LN. Treatment with the new antipsychotic sertindole for lateoccurring undesirable movement effects. Int Clin Psychopharmacol. 2005;20:335-338.

72. Yap YG, Camm J. Risk of torsades de pointes with non-cardiac drugs. Br Med J. 2000;320:1158-1159.

73. Kasper S, Tuomi M. The clinical safety and tolerability profile of sertindole. Int J Neuropsychopharmacology. 2004;7:S422.

74. Mack R, Driscoll R, Silber C. The long term cardiovascular safety of sertindole. Eur Neuropsychopharmacology. 1997;7:S207.

75. Titier K, Canal M, Déridet C, et al. Determination of myocardium to plasma concentration ratios of five antipsychotic drugs: comparison with their ability to induce arrhythmia and sudden death in clinical practice. Toxicol Appl Pharmacol. 2004;199:52-60.

76. Harrigan EP, Miceli JJ, Anziano R, et al. A randomized evaluation of the effects of six antipsychotic agents on QTc, in the absence and presence of metabolic inhibition. J Clin Psychopharmacol. 2004;24: 62-69.
77. DuBuske LM. Second-generation antihistamines: the risk of ventricular arrhythmias. Clin Ther. 1999;21:281-295.

78. Witchel HJ, Hancox JC. Familial and acquired long QT syndrome and the cardiac rapid delayed rectifier potassium current. Clin Exp Pharmacol Physiol. 2000;27:753-766.

79. Glassman AH, Bigger, JT. Antipsychotic drugs: Prolonged QTc interval, torsade de pointes, and sudden death. Am J Psychiatry. 2001;158:1774-1782.

80. Nielsen J, Graff C, Hardahl T, et al. Sertindole causes distinct electrocardiographic T-wave morphology changes. Eur Neuropsychopharmacol. 2009;19:702-707

81. Nielsen J, Andersen MP, Graff C, et al. The effect of sertindole on QTD and TPTE. Acta Psychiatr Scand. 2010;121:385-388.

82. Ray WA, Chung CP, Murray KT, Hall K, Stein CM. Atypical antipsychotic drugs and the risk of sudden cardiac death. $N$ Engl $J$ Med. 2009;360:225-235.

83. Tiihonen J, Lönnqvist J, Wahlbeck K, et al. 11-year follow-up of mortality in patients with schizophrenia: a population-based cohort study (FIN11 study). Lancet. 2009;374:620-627.

84. Spina E, Zoccali R. Sertindole: pharmacological and clinical profile and role in the treatment of schizophrenia. Expert Opin Drug Metab Toxicol. 2008;4(5):629-638.

85. Caccia S, Pasina L, Nobili A. New atypical antipsychotics for schizophrenia: iloperidone. Drug Des Devel Ther. 2010;18;4:33-48.
Drug Design, Development and Therapy

\section{Publish your work in this journal}

Drug Design, Development and Therapy is an international, peerreviewed open-access journal that spans the spectrum of drug design and development through to clinical applications. Clinical outcomes, patient safety, and programs for the development and effective, safe, and sustained use of medicines are a feature of the journal, which

\section{Dovepress}

has also been accepted for indexing on PubMed Central. The manuscript management system is completely online and includes a very quick and fair peer-review system, which is all easy to use. Visit http://www.dovepress.com/testimonials.php to read real quotes from published authors.

Submit your manuscript here: http://www.dovepress.com/drug-design-development-and-therapy-journal 